\title{
Emergent intracranial surgical embolectomy in conjunction with carotid endarterectomy for acute internal carotid artery terminus embolic occlusion and tandem occlusion of the cervical carotid artery due to plaque rupture
}

\author{
Hirotaka Hasegawa, MD, Tomohiro Inoue, MD, Akira Tamura, MD, PhD, and Isamu Saito, MD, PhD \\ Department of Neurosurgery, Fuji Brain Institute and Hospital, Shizuoka, Japan
}

Acute internal carotid artery (ICA) terminus occlusion is associated with extremely poor functional outcomes or mortality, especially when it is caused by plaque rupture of the cervical ICA with engrafted thrombus that elongates and extends into the ICA terminus. The goal of this study was to evaluate the efficacy and safety of surgical embolectomy in conjunction with carotid endarterectomy (CEA) for acute ICA terminus occlusion associated with cervical plaque rupture resulting in tandem occlusion. A retrospective review of medical records was performed. Clinical and radiographic characteristics were evaluated, including details of surgical technique, recanalization grade, recanalization time, complications, modified Rankin Scale (mRS) score at 3 months, and National Institutes of Health Stroke Scale (NIHSS) score improvement at 1 month. Three patients (mean age 77.3 years; median presenting NIHSS Score 22, range 19-26) presented with abrupt tandem occlusion of the cervical ICA and ICA terminus and were selected for surgery after confirmation of embolic high-density signal at the ICA terminus on CT and diffusion-weighted imaging (DWI)/magnetic resonance angiography (MRA) mismatch. All patients underwent craniotomy for surgical embolectomy of the ICA terminus embolus followed by cervical exposure, aspiration of long residual proximal embolus ranging from the cervical to cavernous ICA, and removal of ruptured unstable plaque by CEA. Postoperative MRA demonstrated Thrombolysis In Myocardial Infarction (TIMI) 3 recanalization in all patients (100\%) without evidence of additional infarction according to DWI. Mean recanalization time from hospital arrival was 234 minutes and from start of surgery, 151 minutes. Serial postoperative $\mathrm{CT}$ and MRI studies showed no symptomatic hemorrhage, brain edema, or progression of infarction. The patients' mRS scores at 3 months were 3, 3, and 1. All 3 patients demonstrated marked improvements in NIHSS scores (median 17 points; range 13-23 points) at 1 month. Considering the dismal prognosis associated with ICA terminus occlusion, especially when accompanied by cervical plaque rupture, emergent surgical embolectomy in conjunction with CEA might be an effective and decisive treatment option with a high complete recanalization rate and acceptable safety profile.

http://thejns.org/doi/abs/10.3171/2014.11.JNS132855

KEY WORDS carotid artery stenosis; carotid endarterectomy; carotid terminus occlusion; embolectomy; plaque rupture, vascular disorders

\begin{abstract}
A BRUPT intracranial large-vessel occlusion is associated with poor functional outcomes and a high mortality rate. The only possible solution is restoration of flow as soon as possible within a limited therapeutic time window. ${ }^{1,13,18}$ Among these large vessel occlusions, the internal carotid artery (ICA) terminus occlusion is extremely difficult to manage.
\end{abstract}

Acute ICA terminus occlusion responds poorly to intravenous administration of tissue plasminogen activator (tPA) due to its high clot burden; the recanalization rate is $4.4 \%$ in response to intravenous tPA alone and is $43.5 \%$ in response to intravenous tPA followed by additional endovascular treatment. ${ }^{1}$ Although new endovascular devices have accomplished better outcomes, the recanalization

ABBREVIATIONS ACOA = anterior communicating artery; $C E A=$ carotid endarterectomy; $D W I=$ diffusion-weighted imaging; ICA = internal carotid artery; $M C A=$ middle cerebral artery; MRA = magnetic resonance angiography; $\mathrm{mRS}=$ modified Rankin Scale; NIHSS = National Institutes of Health Stroke Scale; PCoA = posterior communicating artery; TIMI = Thrombolysis In Myocardial Infarction; tPA = tissue plasminogen activator.

SUBMITTED December 25, 2013. ACCEPTED November 13, 2014.

INCLUDE WHEN CITING Published online January 9, 2015; DOI: 10.3171/2014.11.JNS132855.

DISCLOSURE The authors report no conflict of interest concerning the materials or methods used in this study or the findings specified in this paper. 
rate for ICA terminus occlusion remains unsatisfactory, with a relatively high rate of complications and a high mortality rate. ${ }^{3,9,14,15,17,19}$

Most cases of ICA terminus occlusions are caused by cardiogenic embolism (Fig. 1A), although some cases are due to cervical plaque rupture and secondary artery-to-artery large embolus with resultant tandem occlusion of the extracranial ICA and intracranial ICA terminus (Fig. 1B). In such cases, total blockade of anterograde ICA flow and the major collateral pathway of the circle of Willis results in a severe perfusion deficit and acute dense neurological symptoms. Thus, immediate recanalization is mandatory. However, the treatment strategy is extremely difficult because of the coexistence of hard, though fragile, ruptured
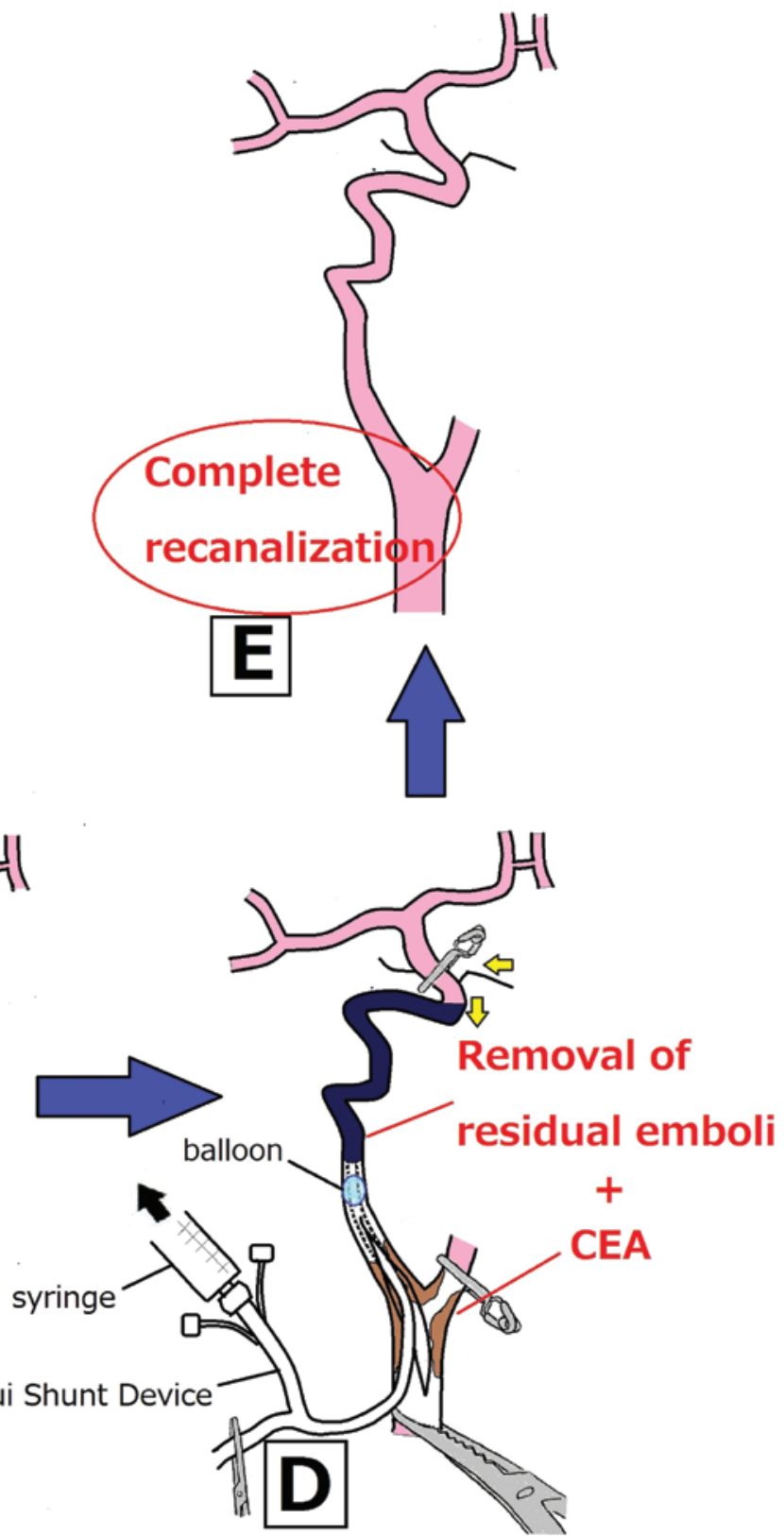
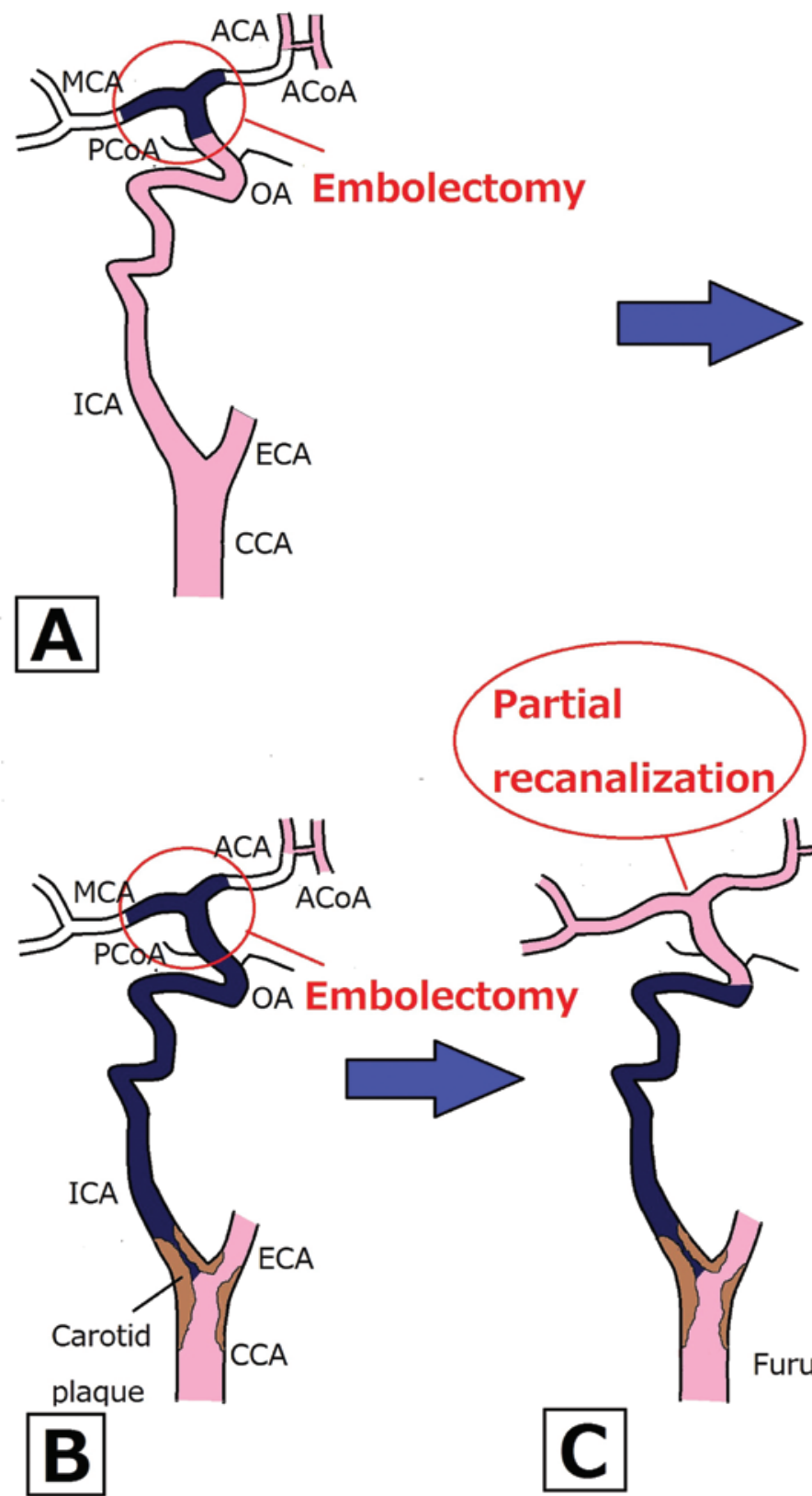

CoA
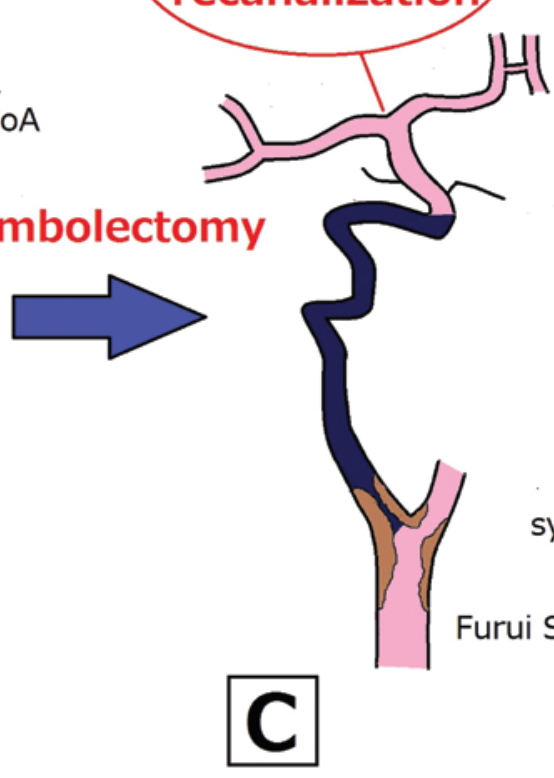

FIG. 1. Schematic drawings of an ICA terminus occlusion (A) and tandem occlusion of cervical ICA/ICA terminus (B) are shown. When the embolus at the ICA terminus is removed, complete recanalization can be achieved in cases of ICA terminus occlusion alone $(A \rightarrow E)$, whereas recanalization is partial and an additional cervical procedure is needed in cases of tandem occlusion $(B \rightarrow C)$. To remove the residual emboli in the ICA, the maneuver using a Furui double-balloon internal shunt system is needed (D). The shunt device is inserted into the proximal ICA, with the tube on the other side of the device clamped, the balloon is inflated within the ICA, and the embolus is aspirated using a 10-ml syringe. The black arrow shows the pressure of the pulling syringe. The yellow arrows show the backflow into the ICA from the ophthalmic artery. The shunt device is removed along with the embolus after deflation of the balloon. This maneuver is based on techniques previously described by McCormick et al. ${ }^{11}$ and Murata et al. ${ }^{12}$ CEA is then performed for removal of the carotid plaque, resulting in complete recanalization (E). Copyright Hirotaka Hasegawa. Published with permission. $\mathrm{ACA}=$ anterior cerebral artery; $\mathrm{CCA}=$ cervical carotid artery; $\mathrm{ECA}=$ external carotid artery; $\mathrm{OA}=$ ophthalmic artery. 
plaque and the secondary engrafted very large thrombi extending from the cervical ICA up to the ICA terminus. To our best knowledge, there is no detailed technical illustration (such as a case report or technical note) to help guide the treatment strategy for this rare but devastating disease.

Since endovascular service is not available in the emergent setting in our institution, we have adopted surgical embolectomy as second-line treatment for occlusion of large intracranial vessels of the anterior circulation based on diffusion-weighted imaging (DWI)/magnetic resonance angiography (MRA) mismatch and have reported fairly good recanalization rates and functional outcomes. ${ }^{6}$ In the present article, we describe 3 cases in which patients underwent emergent surgical embolectomy in conjunction with carotid endarterectomy (CEA) to treat acute tandem occlusion of the cervical ICA and ICA terminus due to cervical plaque rupture and the presence of huge secondary emboli.

\section{Methods \\ Patient Selection}

With institutional review board approval, we retrospectively reviewed the medical charts of 3 consecutive patients who underwent emergent surgical embolectomy in conjunction with CEA for acute ICA terminus occlusion caused by cervical plaque rupture (tandem occlusion of cervical ICA and ICA terminus). The patients' clinical and imaging data were analyzed in detail.

The National Institutes of Health Stroke Scale (NIHSS) score upon initial presentation was determined by the treating physicians (neurosurgical residents). CT and MRI, including DWI, and MRA studies were performed. Informed consent to undergo surgical embolectomy and CEA was obtained from the patient's family in all cases.

Detailed inclusion criteria for emergent surgical embolectomy at our hospital have been reported elsewhere. ${ }^{6}$ In short, the indications were as follows: 1 ) a patient with acute occlusion of the proximal intracranial anterior circulation with NIHSS Score $\geq 15$ or a dense focal deficit, such as severe hemiparesis and/or aphasia; 2) absence of acute hyperintensity involving more than one-third of the middle cerebral artery (MCA) territory on DWI as well as the complete occlusion of unilateral ICA and/or MCA on MRA (so-called DWI/MRA mismatch); 3) a patient who was not eligible for intravenous tPA therapy.

\section{Surgical Embolectomy in Conjunction With CEA}

Under general anesthesia, a standard frontotemporal craniotomy was performed. After introduction of the microscope, the Sylvian fissure was widely dissected to expose the ICA terminus. First, temporary clips were applied to the distal vessels to prevent distal migration of the embolus (Fig. 2A). Then, the embolus was retrieved through a transverse arteriotomy made near its distal end (Fig. 2B). After confirming restored collateral flow via the anterior communicating artery (ACoA) and/or the posterior communicating artery $(\mathrm{PCoA})$ as well as persistent occlusion of the petrous segment of the ICA $\left(\mathrm{C}_{2}\right)$ by microvascular Doppler ultrasonographic assessment, the coexis- tence of the cervical ICA occlusion was recognized (Figs. $1 \mathrm{C}$ and $2 \mathrm{C}$ ). At this point, a temporary clip was applied to the $\mathrm{C}_{2}$ portion of the ICA just proximal to the origin of the PCoA to prevent distal migration of the residual petrous to cavernous emboli and to avoid disturbing the restored collaterals (Fig. 2D).

Then, the cervical carotid artery was exposed. The residual cervical to cavernous ICA embolus was washed out by backflow (Fig. 2E) and/or an aspiration maneuver utilizing a shunt tube. A commercially available trilumen Furui shunt device (Inter Medical Co., Ltd.) ${ }^{5}$ was inserted into the distal ICA, and the distal embolus was aspirated using a 10-ml syringe after inflation of the balloon and temporary clamping of the other side of the shunt tube. The embolus was extracted with the inserted shunt tube after deflation of the balloon (Figs. 1D and 2F and G) following the technique described by McCormick et al. ${ }^{11}$ and Murata et al. ${ }^{12}$ An adequate backflow from the ophthalmic artery was thus obtained. Then, the intracranial temporary clip at the $\mathrm{C}_{2}$ segment was removed. Recanalization was confirmed by flushing normal saline via the shunt tube inserted into the distal ICA, which could be visualized in the intracranial surgical field (Fig. 2H). In addition, after restoration of anterograde flow via the shunt tube, we confirmed robust anterograde flow intracranially using microvascular Doppler. Then, CEA was performed in the usual manner to remove the ruptured unstable plaque (Figs. 1D and 2I), and the arteriotomy was closed in the usual fashion and complete recanalization was obtained (Fig. 1E; see also Videos 1 and 2, which illustrate actual procedures of the urgent surgical embolectomy in conjunction with CEA in cases 3 and 2, respectively).

VIDEO 1. Video clip showing surgical embolectomy with concomitant CEA for abrupt tandem occlusion of the left cervical ICA/ICA terminus in Case 3. Copyright Hirotaka Hasegawa. Published with permission. Click here to view with Media Player. Click here to view with Quicktime.

VIDEO 2. Video clip showing surgical embolectomy with concomitant CEA for abrupt tandem occlusion of the left cervical ICA/ICA terminus in Case 2. Copyright Hirotaka Hasegawa. Published with permission. Click here to view with Media Player. Click here to view with Quicktime.

As anticoagulant treatment, low-molecular-weight heparin (2000-3000 units) was administered intraoperatively just after intracranial embolectomy. Postoperatively single or dual oral antiplatelet therapy and statins as well as diabetes medicines were administered. Blood pressure was strictly controlled below $130 \mathrm{~mm} \mathrm{Hg}$ by intravenous infusion of a calcium blocker during the first few postoperative days.

\section{Postoperative Evaluation and Follow-Up}

CT scans were performed several times to assess for hemorrhagic complications or new ischemic lesions during the acute period, including immediately after surgery, on postoperative Day 1, and every few days during the first 2 weeks. Follow-up MRI studies, including MRA and DWI, were performed to determine the recanalization status and to assess any new ischemic lesions immediately after surgery on Day 1 (short-term), after 1 week, and after 1 month (long-term) or later. Final recanalization status was assessed based on MRA performed immediately after sur- 

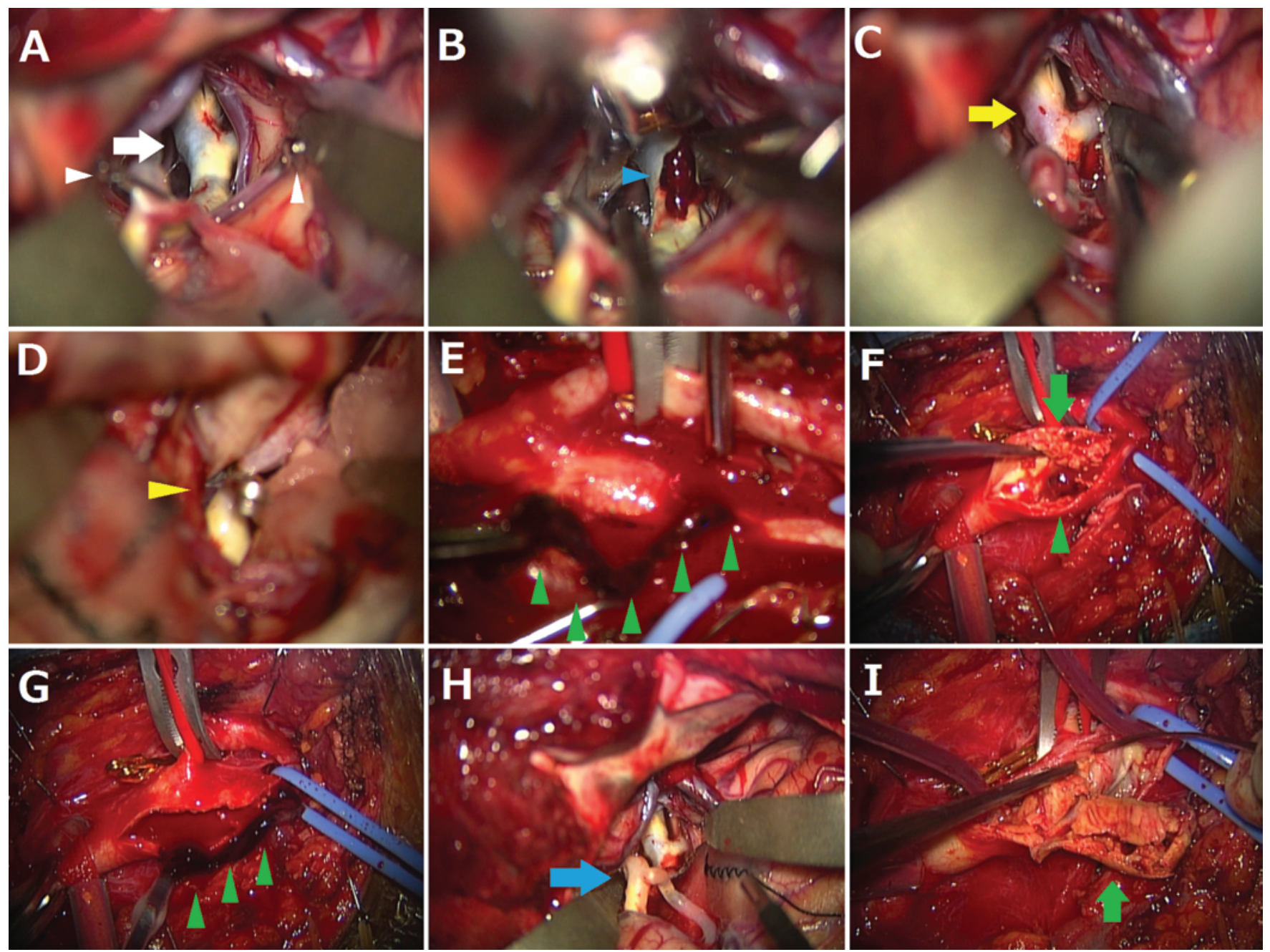

FIG. 2. Representative intraoperative photographs (all left-side images) from Case 1 (A-D and F-H) and Case 3 (E). Bluish discoloration at the occluded ICA terminus suggested the location of embolus (white arrow) (A). Temporary clips were applied to the distal vessels (in this case, the $\mathrm{M}_{1}$ segment of the MCA and the anterior temporal artery, white arrowheads) to prevent distal migration of the embolus. The embolus (blue arrowhead) was then removed through a transverse arteriotomy made around the distal end of the embolus (B). After removal of the embolus, partial recanalization via collateral circulation from the circle of Willis was confirmed by microvascular Doppler assessment. However, flow signal of $C_{2}$ was not confirmed, which suggested occlusion of the cervical ICA. The change in color of the ICA terminus from bluish to reddish suggested only partial but significant revascularization (yellow arrow) (C). After confirmation of cervical ICA occlusion, a temporary clip (yellow arrowhead) was applied to the ICA just proximal to the PCoA, preserving collateral flow (D). In Case 3 (only), the residual intraarterial embolus $(\sim 10-\mathrm{cm}$ embolus, green arrowheads) was washed out by backflow from the ophthalmic artery after creation of an arteriotomy at the cervical ICA (E), whereas in Cases 1 and 2, the embolus was removed with the shunt device and a 10-ml syringe as described below. In Cases 1 and 2, the cervical carotid artery was exposed, and a severe atherosclerotic plaque (green arrow) with residual intraarterial embolus was seen through the arteriotomy made at the ICA origin (F). A trilumen internal shunt device was inserted into the distal ICA, and the residual embolus (green arrowheads) was aspirated using a 10-ml syringe and extracted with the inserted shunt tube (G). The recanalization was confirmed by flushing normal saline via the shunt tube inserted into the distal ICA, which was visualized in the intracranial surgical field (H). The blue arrow shows normal saline reaching the MCA bifurcation. CEA was performed, and a large atherosclerotic plaque with severe intraluminal stenosis was removed (green arrow) (I).

gery according to the Thrombolysis In Myocardial Infarction (TIMI) system. ${ }^{21}$ Progression of ischemic lesions was evaluated by MRI-DWI. Symptomatic hemorrhagic complication was defined as CT evidence of new intracranial hemorrhage with apparent neurological worsening of at least 4 points on the NIHSS scale, and symptomatic brain edema was determined using the same criteria. Functional outcomes were evaluated at approximately 1 month (NIHSS score) and at approximately 3 months (modified
Rankin Scale [mRS] score) via a review of medical records or by telephone interview of the patient's family.

\section{Results \\ Overall Results}

Between March 2008 and October 2013, 1737 consecutive patients with acute ischemic stroke were admitted to and treated at our hospital. Among them, 3 patients 
TABLE 1. Summary of demographic and clinical characteristics of 3 cases involving patients who underwent embolectomy and concomitant CEA

\begin{tabular}{|c|c|c|c|}
\hline Characteristic & Case 1 & Case 2 & Case 3 \\
\hline Age (years) & 77 & 84 & 71 \\
\hline Sex & Male & Male & Male \\
\hline Presenting NIHSS score & 26 & 22 & 19 \\
\hline Affected side & Left & Left & Left \\
\hline No. of atherosclerotic risk factors & 2 (HTN, DL) & 3 (HTN, DM, smoking) & 3 (HTN, DL, smoking) \\
\hline Contralateral ICA stenosis & Yes & Yes & Yes \\
\hline Intracranial atherosclerosis & No & Yes & Yes \\
\hline Atrial fibrillation & No & No & No \\
\hline Final recanalization status (TIMI grade) & 3 & 3 & 3 \\
\hline Time to start of surgery (minutes)* & 80 & 74 & 95 \\
\hline Partial† recanalization time (minutes) $\ddagger$ & 70 & 60 & 36 \\
\hline Complete recanalization time (minutes) $\ddagger$ & 236 & 136 & 81 \\
\hline Symptomatic complications & No & No & No \\
\hline Progress of infarction & No & No & No \\
\hline NIHSS score at 1 mo postop & 3 & 9 & 2 \\
\hline mRS score at 3 mos postop & 3 & 3 & 1 \\
\hline $\begin{array}{l}\mathrm{DL}=\text { dyslipidemia; } \mathrm{DM}=\text { diabetes mellitus; } \mathrm{HTN}= \\
\text { * Time from hospital arrival. } \\
\dagger \text { Recanalization of ICA terminus via collateral flon } \\
\text { † Recanalization time from start of surgery. }\end{array}$ & cle of Willis. & & \\
\hline
\end{tabular}

$(0.17 \%)$ were diagnosed with ICA terminus occlusion due to cervical plaque rupture and underwent surgical embolectomy in conjunction with CEA. Patient data are summarized in Table 1. The mean presenting NIHSS score was 22.3 points. At presentation, CT demonstrated a high signal density at the occluded ICA terminus in all patients. Subsequent emergent MRA confirmed a signal defect in the occluded ICA area, and DWI revealed a limited area of hyperintensity, usually around the external capsule and insula, in all patients. The time of onset of occlusion was unknown in all 3 patients, and thus, intravenous tPA therapy was contraindicated. Therefore, surgical embolectomy was selected as a second-line treatment.

The mean recanalization time from hospital arrival and from start of surgery was 234 minutes and 151 minutes, respectively. MRA performed just postoperatively confirmed TIMI 3 recanalization in all patients (100\%). Mid-term to long-term patency was also confirmed by MRA (mean 1.3 months, range 1-2 months). No patient developed significant stenosis at the surgical site during the follow-up period.

All patients had slight asymptomatic subarachnoid hemorrhage near the operation site attributable to minor pial injury during surgical manipulation. However, there was no parenchymal hematoma or confluent additional infarction causing neurological worsening, as confirmed by postoperative serial CT and MRI studies. Imaging findings from cases 1, 2, and 3 are demonstrated in Figs. 3, 4, and 5, respectively. All patients demonstrated marked neurological improvement, as indicated by improvements in NIHSS score at 1 month (mean 17 points, range 13-23 points). The mRS scores at 3 months were 3,3 , and 1, respectively.
Postoperative investigation by a cardiologist excluded the risk factors for cardiac embolism. In addition, all patients had several atherosclerotic risk factors. Retrieved clots and cervical plaque from the patients in cases 1 and 3 were examined histologically, revealing unstable ruptured atherosclerotic cervical plaque as well as engrafted secondary thrombus.

\section{Case Illustration}

\section{Case 3}

This 71-year-old man was emergently referred to our hospital due to altered mental status, right hemiplegia, and global aphasia. His presenting NIHSS score was 19. Initial CT showed a left hyperdense ICA terminus and MCA sign (Fig. 5A). MRA demonstrated a left ICA occlusion, but ischemic lesions on DWI were limited to the basal ganglia (Fig. 5B-D). Electrocardiography did not show atrial fibrillation. With the intracranial procedure, recanalization of the ICA terminus and the MCA was obtained at 131 minutes after hospital arrival and at 36 minutes after the start of surgery. With the additional CEA, complete recanalization was obtained at 176 minutes after hospital arrival and at 81 minutes after the start of surgery. MRI obtained immediately after the operation showed TIMI 3 recanalization without additional ischemic lesions on DWI (Fig. 5F-I). A CT scan performed on postoperative Day 1 showed slight subarachnoid hemorrhage around the surgical field, but the patient remained asymptomatic (Fig. $5 \mathrm{E})$. His level of consciousness, aphasia, and hemiplegia markedly improved. At 1 month, his NIHSS score was 2, with only residual slight right weakness and slight aphasia, and he was transferred to the rehabilitation unit at our 

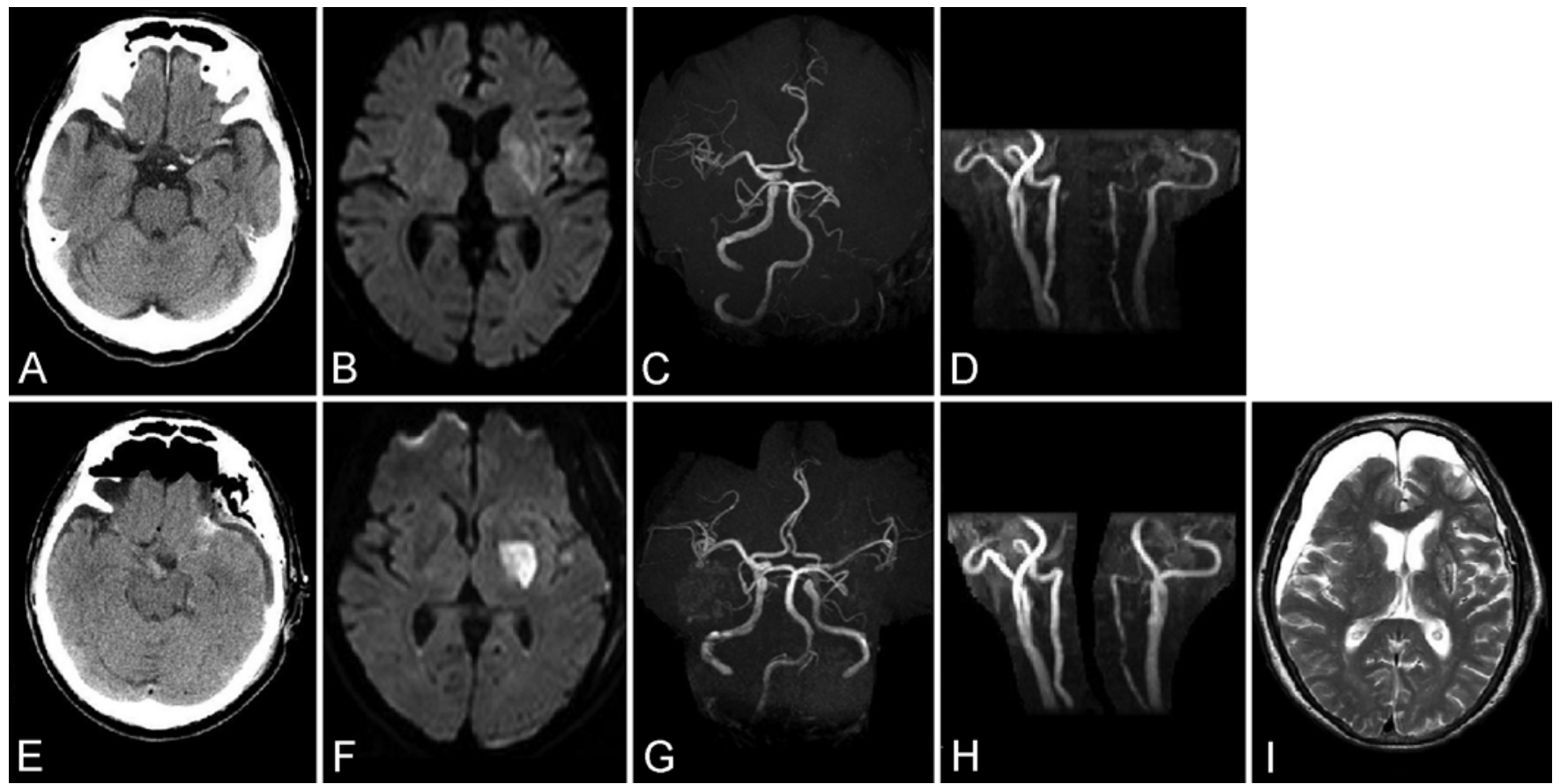

FIG. 3. Imaging studies from Case 1. Emergent CT revealed a high-density sign at the ICA terminus as well as at the proximal MCA (A). Although the ischemic lesions on DWI were limited to the basal ganglia and a small area of the cortex (B), MRA of the head (C) and neck (D) showed absence of flow signal. Urgent surgical revascularization with embolectomy plus CEA was successfully performed, and CT (E), DWI (F), and MRA on postoperative Day 1 revealed TIMI 3 recanalization and no additional ischemic lesions with slight asymptomatic subarachnoid hemorrhage around the surgical area. At 1 month after the surgery, MRA showed good patency of the left ICA and MCA (G and H), and T2-weighted MRI showed a small high-intensity area compatible with old infarction at the left basal ganglia (I).

institution. At 3 months, he could ambulate without apparent motor deficit and was discharged home; his final mRS score was 1.

\section{Discussion}

The present study demonstrates that emergent surgical embolectomy in conjunction with CEA for ICA terminus embolic occlusion associated with carotid plaque rupture can accomplish complete recanalization with an acceptable safety profile.

Among the intracranial acute large vessel occlusions, ICA terminus occlusion is associated with by far the poorest functional outcome and the highest mortality rate. ${ }^{1}$ When it is caused by cervical plaque rupture, very large emboli derived from the engrafted thrombus on the ruptured plaque can occlude all the way from the cervical ICA up to the ICA terminus, and thus definite and decisive flow restoration treatment is mandatory.

Considering the extremely high clot burden, intravenous tPA has little efficacy for emergent recanalization in the context of ICA terminus occlusion. ${ }^{1}$ Endovascular devices, including the Merci Retriever (Stryker), the Penumbra System (Penumbra, Inc.), and the Solitaire FR (ev3-Covidien) are in current use. ${ }^{9,17,18}$ In a single-center experience with the Penumbra System, partial or complete reperfusion was achieved in $86 \%$ of ICA terminus occlusions, ${ }^{15}$ and the Solitaire FR is expected to accomplish even better recanalization. ${ }^{3,14,17}$ However, when we focus on the complete recanalization only, rates are only $40 \%-50 \%$ of all treated patients. ${ }^{3,9,14,15,17}$ Considering that these reports mainly focused on pure embolic ICA terminus occlusion rather than tandem occlusion, the expected complete recanalization rate for tandem occlusions (ICA terminus plus cervical ICA occlusion) would be even lower.

Some authors have focused on endovascular treatment of tandem extracranial/intracranial occlusion, mostly dealing with tandem occlusion of the cervical ICA and intracranial MCA, which is apparently associated with a lower clot burden..$^{2,4,7,8,20}$ Very few articles deal with cases of tandem cervical ICA/ICA terminus occlusion, and most of those articles focus primarily on tandem cervical ICA/ MCA occlusion or acute occlusion of other main cerebral arterial trunks. Most of the articles discuss the difficulty of treatment of tandem occlusion and suggest the feasibility of extracranial (cervical) ICA revascularization by stent placement as the first step. We believe that among all the tandem occlusions, cervical ICA/ICA terminus occlusion should be regarded as by far the most devastating, because it is derived from cervical plaque rupture and very large secondary emboli that extend up to the ICA terminus and thus obstruct all possible major collateral pathways. In fact, the largest case series focusing on tandem occlusion, which was reported by Malik et al., demonstrated significantly higher recanalization rates of tandem cervical ICA/MCA occlusion compared with tandem cervical ICA/ICA terminus occlusion (43 [84.3\%] of 51 cases vs 15 [57.7\%] of 26 cases, $p=0.02) .{ }^{10}$ Considering the successful (both complete and partial) recanalization rates of $57.7 \%$ (15 of 26 cases) in this series of tandem cervical ICA/ICA 

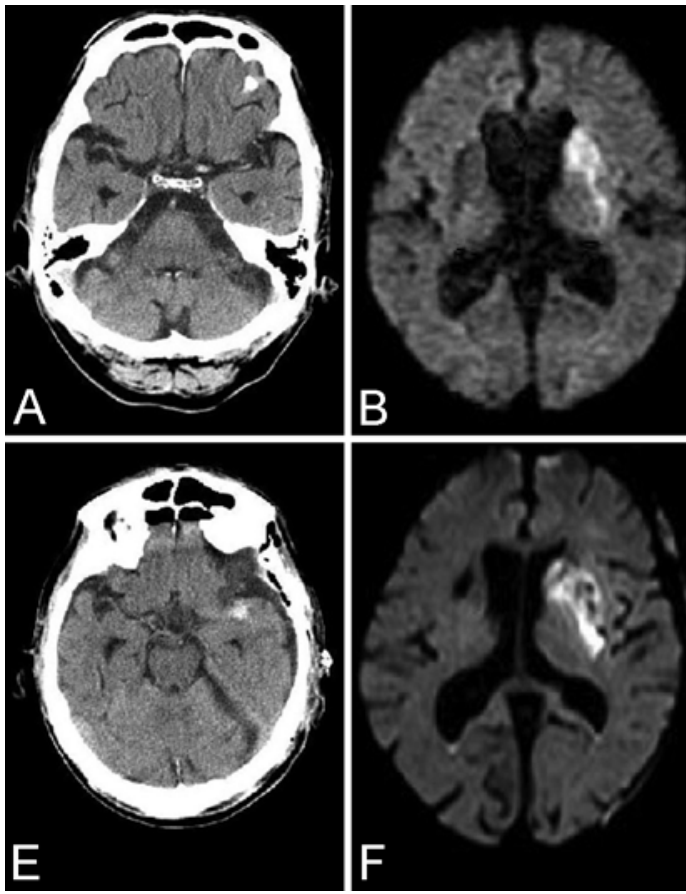
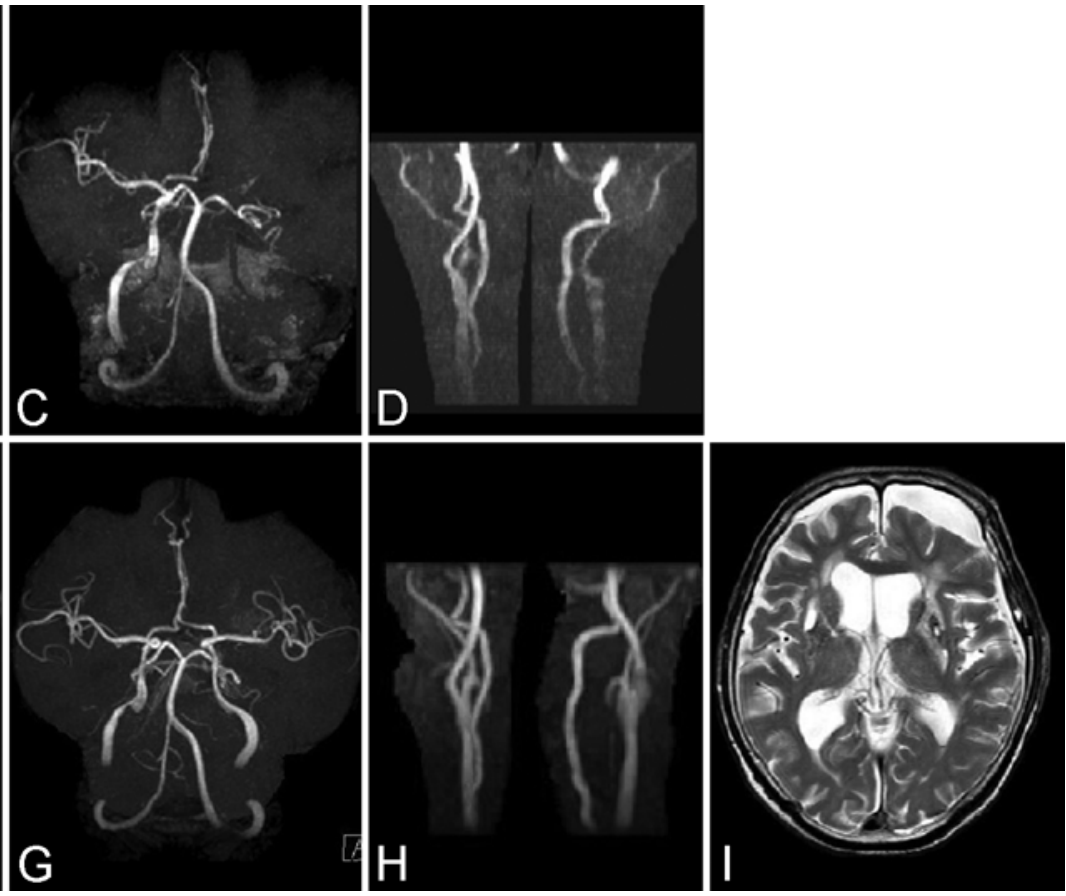

FIG. 4. Imaging studies from Case 2. Emergent CT revealed a high-density sign at the left ICA terminus and at the MCA (A). MRA showed left ICA terminus occlusion ( $C$ and $\mathbf{D})$, but ischemic lesions on DWI were limited to the basal ganglia (B). Emergent surgical embolectomy and CEA was performed, and MRI immediately after the operation (F) and at 1 month postoperatively (G-I) showed TIMI 3 recanalization with no additional ischemic lesions on DWI and T2-weighted MRI. CT on postoperative Day 1 showed slight subarachnoid hemorrhage around the surgical field, but the patient remained asymptomatic (E).

terminus occlusions, we could expect an even lower complete recanalization rate. This presumably quite low rate of complete recanalization in response to endovascular in- tervention in cases of cervical and ICA terminus tandem occlusion might be due to several factors. For example, all endovascular mechanical embolectomy devices need to
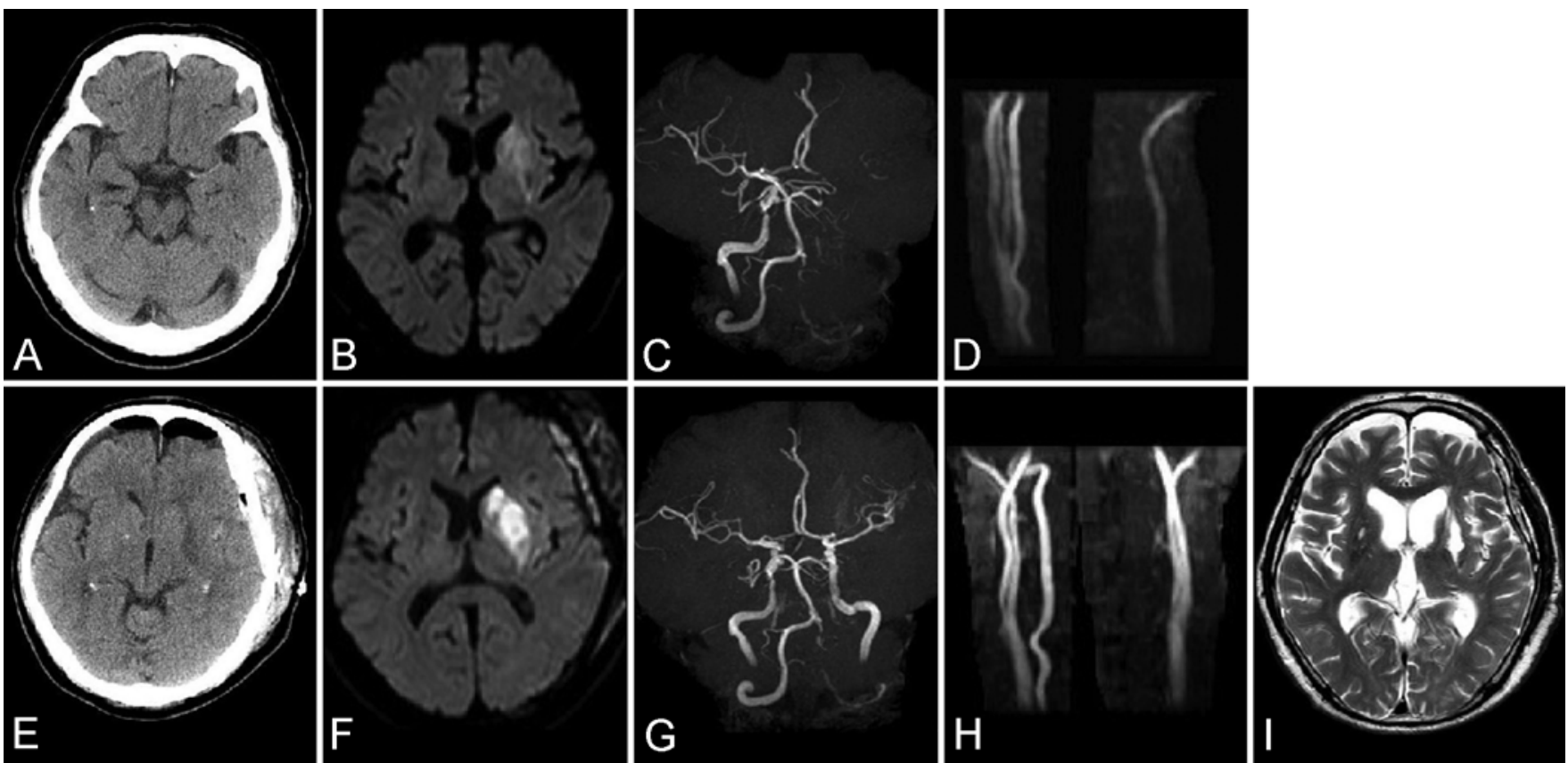

FIG. 5. Imaging studies from Case 3. Initial CT showed a left hyperdense ICA terminus and MCA sign (A), and MRA demonstrated a left ICA occlusion (C and D), but ischemic lesions on DWI were limited to the basal ganglia (B). MRI studies performed immediately after the operation (F) and 2 months postoperatively (G-I) showed TIMI 3 recanalization without additional ischemic lesions on DWI and T2-weighted MRI, although slight subarachnoid hemorrhage around the surgical field was noted on CT images obtained on postoperative Day 1 (E). 
be deployed through unstable carotid plaque; thus, distal migration of emboli cannot be avoided completely despite use of a distal protection device or proximal flow control strategy. Even with stenting procedures to stabilize the extremely unstable cervical plaque, mid to late restenosis or late distal embolization is another concern.

Therefore, the use of decisive and durable (although invasive) surgical embolectomy plus CEA should be considered to overcome this unfavorable condition. In the present series, all 3 patients were successfully treated, and TIMI 3 recanalization rates were $100 \%$ (3 of 3 cases) with excellent mid- to long-term patency. The final revascularization status is the strongest predictor of good outcome in the context of acute large-vessel occlusion. ${ }^{1,13,18}$ Accordingly, marked neurological improvement was obtained in all patients in this series. One of the advantages of direct surgical embolectomy when compared with endovascular methods is immediate distal clip application to prevent distal embolism during the procedure, which definitely contributes to the establishment of complete recanalization. ${ }^{6}$

Appropriate patient selection is critical when considering safe, effective, and aggressive treatments for this condition. In this series, we used DWI/MRA mismatch to select patients, and no significant reperfusion injury (e.g., intraparenchymal hemorrhage or brain swelling) occurred. Recent studies have suggested a relationship between initial DWI volume on clinical outcome after recanalization and avoidance of therapeutic complications. ${ }^{13}$ In other words, the area of initial DWI hyperintensity could represent the net cumulative result of acute ischemic impact by large vessel occlusion and the extent and rescue effect of the collateral circulation.

The time needed for recanalization treatment is also an important issue. In the present series, every effort was made to minimize time loss, as mentioned previously. ${ }^{6}$ First, the patients were directly transported from the MRI suite to the operating room without diagnostic angiography, and the time required from hospital arrival to initiation of surgery was 83 minutes on average. Second, standard frontotemporal craniotomy with minimal hemostasis required less than 20 minutes, and we were able to accomplish the first partial recanalization (via the ACoA by the contralateral ICA and/or the PCoA by the posterior circulation toward the ipsilateral MCA area) in an average of 138 minutes from hospital arrival (Table 1), which compares favorably with the time to recanalization associated with endovascular methods.

If good clinical outcome is defined as an mRS score of $0-2$, then $33 \%$ of the patients ( 1 of 3 ) in this series had a good outcome, which might be low when compared with the rates seen in most studies focusing on acute large vessel occlusions as a whole. The occluded vessel was on the left side in all patients in our series; thus, even after successful recanalization and significant motor improvement, residual mild aphasia and mild right hemiparesis tended to make the mRS score worse. Indeed, the patients in both Case 1 and Case 2 had an mRS score of 3 at the 3-month postoperative assessment. Although they were ambulatory, they remained dependent in daily life due to mild aphasia. Among large-vessel occlusions, abrupt ICA terminus occlusion is quite devastating. ${ }^{16}$ Thus, not only the final mRS score but also the range of functional improvement from initial dense neurological deficit should be evaluated in such cases. We investigated the NIHSS score at 1 month, which showed marked improvements in all cases (17-point increase on average). In addition, there were no deaths within 90 days of surgery in this series. From the previous articles dealing with recanalization therapy of acute largevessel occlusions, mortality rates were $10 \%-50 \%$, which suggests that our results were favorable. ${ }^{3,9,14,15,17,18}$

The main limitation of our study derives from its retrospective nature and small patient number. However, to date, therapeutic strategies of cervical ICA/ICA terminus tandem occlusion have been mainly based on small case series. Additional cases should be accumulated.

\section{Conclusions}

Under conditions of patient selection based on DWI/ MRA mismatch, emergent surgical embolectomy in conjunction with CEA was associated with a high complete recanalization rate as well as a good safety profile in patients with abrupt ICA terminus occlusion due to cervical plaque rupture. This decisive (albeit invasive) approach might be a powerful alternative to endovascular mechanical embolectomy, considering the endovascular difficulty in reopening unstable ruptured plaque and retrieving long emboli without producing distal migration. The otherwise poor prognosis of this disease may warrant this aggressive but effective approach.

\section{References}

1. Bhatia R, Hill MD, Shobha N, Menon B, Bal S, Kochar P, et al: Low rates of acute recanalization with intravenous recombinant tissue plasminogen activator in ischemic stroke: realworld experience and a call for action. Stroke 41:2254-2258, 2010

2. Dababneh H, Guerrero WR, Khanna A, Hoh BL, Mocco J: Management of tandem occlusion stroke with endovascular therapy. Neurosurg Focus 32(5):E16, 2012

3. Dávalos A, Pereira VM, Chapot R, Bonafé A, Andersson T, Gralla J: Retrospective multicenter study of Solitaire FR for revascularization in the treatment of acute ischemic stroke. Stroke 43:2699-2705, 2012

4. Fischer U, Mono ML, Schroth G, Jung S, Mordasini P, ElKoussy M, et al: Endovascular therapy in 201 patients with acute symptomatic occlusion of the internal carotid artery. Eur J Neurol 20:1017-1024, e87, 2013

5. Furui T, Hasuo M: Indwelling double-balloon shunt for carotid endarterectomy. Technical note. J Neurosurg 60:861-863, 1984

6. Inoue T, Tamura A, Tsutsumi K, Saito I, Saito N: Surgical embolectomy for large vessel occlusion of anterior circulation. Br J Neurosurg 27:783-790, 2013

7. Jovin TG, Gupta R, Uchino K, Jungreis CA, Wechsler LR, Hammer MD, et al: Emergent stenting of extracranial internal carotid artery occlusion in acute stroke has a high revascularization rate. Stroke 36:2426-2430, 2005

8. Kim DJ, Kim DI, Byun JS, Jung JY, Suh SH, Kim EY, et al: Intra-arterial thrombolytic therapy for hyperacute ischemic stroke caused by tandem occlusion. Cerebrovasc Dis 26:184-189, 2008

9. Kulcsár Z, Bonvin C, Pereira VM, Altrichter S, Yilmaz H, Lövblad KO, et al: Penumbra system: a novel mechanical thrombectomy device for large-vessel occlusions in acute stroke. AJNR Am J Neuroradiol 31:628-633, 2010 
10. Malik AM, Vora NA, Lin R, Zaidi SF, Aleu A, Jankowitz BT, et al: Endovascular treatment of tandem extracranial/intracranial anterior circulation occlusions: preliminary singlecenter experience. Stroke 42:1653-1657, 2011

11. McCormick PW, Spetzler RF, Bailes JE, Zabramski JM, Frey JL: Thromboendarterectomy of the symptomatic occluded internal carotid artery. J Neurosurg 76:752-758, 1992

12. Murata T, Horiuchi T, Nitta J, Sakai K, Ogiwara T, Kobayashi $\mathrm{S}$, et al: Urgent open embolectomy for cardioembolic cervical internal carotid artery occlusion. Neurosurg Rev 33:341348,2010

13. Olivot JM, Mosimann PJ, Labreuche J, Inoue M, Meseguer E, Desilles JP, et al: Impact of diffusion-weighted imaging lesion volume on the success of endovascular reperfusion therapy. Stroke 44:2205-2211, 2013

14. Pereira VM, Gralla J, Dávalos A, Bonafé A, Castaño C, Chapot R, et al: Prospective, multicenter, single-arm study of mechanical thrombectomy using Solitaire Flow Restoration in acute ischemic stroke. Stroke 44:2802-2807, 2013 (Erratum in Stroke 44:e239, 2013)

15. Psychogios MN, Kreusch A, Wasser K, Mohr A, Gröschel K, Knauth M: Recanalization of large intracranial vessels using the penumbra system: a single-center experience. AJNR Am J Neuroradiol 33:1488-1493, 2012

16. Saqqur M, Uchino K, Demchuk AM, Molina CA, Garami Z, Calleja S, et al: Site of arterial occlusion identified by transcranial Doppler predicts the response to intravenous thrombolysis for stroke. Stroke 38:948-954, 2007

17. Saver JL, Jahan R, Levy EI, Jovin TG, Baxter B, Nogueira $\mathrm{RG}$, et al: Solitaire flow restoration device versus the Merci Retriever in patients with acute ischaemic stroke (SWIFT): a randomised, parallel-group, non-inferiority trial. Lancet 380:1241-1249, 2012

18. Shi ZS, Loh Y, Walker G, Duckwiler GR: Clinical outcomes in middle cerebral artery trunk occlusions versus secondary division occlusions after mechanical thrombectomy: pooled analysis of the Mechanical Embolus Removal in Cerebral Ischemia (MERCI) and Multi MERCI trials. Stroke 41:953960, 2010

19. Smith WS, Sung G, Saver J, Budzik R, Duckwiler G, Liebeskind DS, et al: Mechanical thrombectomy for acute ischemic stroke: final results of the Multi MERCI trial. Stroke 39:1205-1212, 2008
20. Thomalla G, Kruetzelmann A, Siemonsen S, Gerloff C, Rosenkranz M, Röther J, et al: Clinical and tissue response to intravenous thrombolysis in tandem internal carotid artery/middle cerebral artery occlusion: an MRI study. Stroke 39:1616-1618, 2008

21. TIMI Study Group: The Thrombolysis in Myocardial Infarction (TIMI) trial. Phase I findings. N Engl J Med 312:932936, 1985

\section{Author Contributions}

Conception and design: Hasegawa, Inoue. Acquisition of data: Hasegawa. Analysis and interpretation of data: Hasegawa. Drafting the article: Hasegawa. Critically revising the article: all authors. Reviewed submitted version of manuscript: all authors. Approved the final version of the manuscript on behalf of all authors: Hasegawa. Administrative/technical/material support: Inoue. Study supervision: Inoue, Tamura, Saito.

\section{Supplemental Information \\ Videos}

Video 1, Media Player. http://mfile.akamai.com/21490/wmv/ digitalwbc.download.akamai.com/21492/wm.digitalsource-naregional/jns13-2855_video_1.asx.

Video 1, Quicktime. http://mfile.akamai.com/21488/mov/ digitalwbc.download.akamai.com/21492/qt.digitalsource-global/ jns13-2855_video_1.mov.

Video 2, Media Player. http://mfile.akamai.com/21490/wmv/ digitalwbc.download.akamai.com/21492/wm.digitalsource-naregional/jns13-2855_video_2.asx.

Video 2, Quicktime. http://mfile.akamai.com/21488/mov/ digitalwbc.download.akamai.com/21492/qt.digitalsource-global/ jns13-2855_video_2.mov.

\section{Correspondence}

Hirotaka Hasegawa, Department of Neurosurgery, Fuji Brain Institute and Hospital, 270-12 Sugita, Fujinomiya, Shizuoka 4180021, Japan. email: ayhi_hase_dani@yahoo.co.jp. 